\title{
Comparison of the Effects of Sufentanil and Fentanyl on Postoperative Sleep Quality of Children Undergoing Tonsillectomy and Adenotomy: A Randomized Controlled Trial
}

\author{
Yang $\mathrm{Li}^{1, *}$ \\ Bijia Song ${ }^{2, *}$ \\ Zhen $\mathrm{Li}^{3}{ }^{3}$ \\ Jingjie Wan ${ }^{4}$ \\ Man Luo ID' \\ Wenxin Wei (D) \\ Junchao Zhu' \\ 'Department of Anesthesiology, \\ Shengjing Hospital of China Medical \\ University, Shenyang, Liaoning, People's \\ Republic of China; ${ }^{2}$ Department of \\ Anesthesiology, Friendship Hospital of \\ Capital Medical University, Beijing, \\ People's Republic of China; ${ }^{3}$ Department \\ of Anesthesiology and Perioperative \\ Medicine, Shanghai Fourth People's \\ Hospital Affiliated to Tongji University \\ School of Medicine, Shanghai, People's \\ Republic of China; ${ }^{4}$ Department of \\ Anesthesiology, First Affiliated Hospital \\ of Suzhou University, Suzhou, People's \\ Republic of China
}

*These authors contributed equally to this work

\begin{abstract}
Objective: The primary purpose was to compare the effects of sufentanil and fentanyl on the postoperative sleep quality. And the secondary purposes were to evaluate perioperative hemodynamics, postoperative pain and complications of children undergoing tonsillectomy and adenotomy.

Methods: Seventy-six patients were randomly assigned to the sufentanil or fentanyl group. The subjective sleep quality was assessed by the Athens Insomnia Scale (AIS) on the night before surgery (Sleep preop 1), the first night after surgery (Sleep POD 1), and the third night after surgery (Sleep POD 3). The Faces Pain Scale-Revised (FPS-R) was used to evaluate the postoperative pain level 24 hours after surgery. The Observer's Assessment of Alertness and Sedation (OAA/S) scale was used to assess the level of sedation in children. Perioperative hemodynamics and adverse effects were also evaluated.

Results: The AIS score in the sufentanil group was significantly lower at Sleep POD 1 and Sleep POD 3 ( $\mathrm{P}<0.001$, respectively). Children in the sufentanil group had significantly lower FPS-R scores at 2, 4, and 6 hours after surgery $(\mathrm{P}=0.004, \mathrm{P}=0.004$, and $\mathrm{P}=0.001$, respectively). The intraoperative hemodynamic parameters were more stable $(\mathrm{P}<0.05$, respectively) and the OAA/S scores at 2 hours after surgery were lower in the sufentanil group $(\mathrm{P}<0.05)$. There was no significant difference in the incidence of postoperative nausea and vomiting between the two groups $(\mathrm{P}=0.435)$.

Conclusion: Children undergoing tonsillectomy and adenotomy after general anesthesia who received sufentanil had better postoperative sleep quality and less postoperative pain at 2, 4, and 6 hours post operation. Moreover, children who received sufentanil showed better hemodynamic stability during surgery. Therefore, sufentanil should be considered as a better choice to facilitate rapid recovery in children following tonsillectomy and adenotomy.
\end{abstract}

Keywords: postoperative sleep quality, pain, sufentanil, fentanyl, children

\section{Introduction}

Adenoidectomy and tonsillectomy are the most common operations performed in children. A previous study has shown that up to $85 \%$ of children undergoing surgery will experience severe postoperative pain. ${ }^{1}$ Furthermore, most of these children experience pain after being discharged from the hospital, during the recovery phase at home. ${ }^{2}$ Due to postoperative pain, $26 \%$ of children showed postoperative agitation, which increased the difficulty of mask ventilation and
Correspondence: Junchao Zhu Department of Anesthesiology, Shengjing Hospital of China Medical University, Shenyang, Liaoning, People's Republic of China

Tel +8618940257257

Email zhujunchaol@hotmail.com 
bleeding from the surgical site, thus resulting in short-term airway obstruction and hypoxemia. ${ }^{3}$ In addition to these complications, children with severe postoperative pain are more likely to have behavioral delays, poor postoperative sleep quality, and delayed clinical recovery. ${ }^{4}$ And pediatric patients who experienced postoperative pain reported sleep disturbances. ${ }^{5}$ Due to younger children cannot communicate their subjective feelings of pain well, the postoperative sleep status of children can be a good indicator of their postoperative feelings. Poor sleep may be characterized by difficulty falling or staying asleep, poor sleep quality, short sleep duration, and/or poor sleep hygiene. ${ }^{6}$ Although opioids are widely used analgesics, evidence suggests that they may also impair sleep and sleep architecture. ${ }^{7}$ A high dose of opioids could disrupt sleep by reducing rapid eye movement sleep and stage N3 sleep, and increasing wakefulness. ${ }^{7}$ Fentanyl is commonly administered to children undergoing adenotonsillectomy, owing to its high lipid solubility and potency. ${ }^{8-10}$ Sufentanil is an analog of fentanyl that has also been used to induce and maintain anesthesia in children. ${ }^{11}$ Sufentanil is more potent than fentanyl and is the most effective opioid analgesic used in clinical practice. ${ }^{12,13}$ Previous studies have shown that sufentanil might be beneficial over fentanyl by relieving pain and reducing the incidence of opioidrelated adverse events. ${ }^{14,15}$ However, these studies were mostly performed in adults. ${ }^{14,16,17}$ Based on this consideration, this randomized controlled trial was designed to compare the effects of sufentanil and fentanyl on the postoperative sleep quality of children undergoing pediatric tonsillectomy and adenotomy.

\section{Materials and Methods}

This study was approved by the Human Research Ethical Committee of Shengjing Hospital (Shenyang, China; IRB registration no. 2016PS281K) and complied with the Declaration of Helsinki. Written informed consent was obtained from all the patient's parents/guardians. The trial was registered prior to patient enrollment (ChiCTR1800014302).

Inclusion criteria were as follows: children aged 4-12 years with an American Society of Anesthesiologists (ASA) classification of I or II. Exclusion criteria were as follows: recent symptoms of inflammation in the upper respiratory tract, obvious cardio-pulmonary function abnormalities, history of psychiatric diseases, severe developmental disorders or abnormal airways, other neurological impairments such as paralysis or demyelinating diseases, sensitive skin, allergy to propofol or opioids, or abnormal hepatic or renal functions.

\section{Sample Size}

Based on the primary outcome between the two groups in our preliminary study and according to the calculation of the sample size $\left(\mathrm{n}_{1}=\mathrm{n}_{2}=2 *[(1.96+0.842) * \delta / \sigma]^{2}\right)$, we chose 0.55 as the estimated variability between the two groups, and 0.8 as the standard deviation. Therefore, 33 patients were required for each group, assuming a two-sided Type I error $(\alpha)$ of 0.05 and a power of $80 \%$. Potential loss was expected during follow-up or due to drop out; therefore, a total of 76 patients were enrolled in this study.

\section{Study Protocol and Standardized Anesthesia}

Ninety-three patients who were planning to undergo elective tonsillectomy and adenotomy after receiving general anesthesia in Shengjing Hospital of China Medical University were selected. After excluding 17 patients, 76 patients were randomly assigned either to the sufentanil (Group S, n=38) or fentanyl (Group F, n=38) anesthesia group in a 1:1 ratio using a computer-generated randomization number sequence. The group assignment was sealed in sequentially numbered opaque envelopes. All the children fasted overnight, and oral intake of clear fluids was restricted for 2-3 hours before surgery. Patients were taken to the preoperative preparation room with their parents/ guardians approximately 30 minutes prior to surgery. After entering the operating room, all children received standard anesthesia monitoring, consisting of electrocardiogram, noninvasive blood pressure, pulse oxygen saturation, capnography, inspired and end-tidal concentrations of oxygen. After routine pre-oxygenation for 3 minutes, children in Group S were induced with propofol $2.0 \mathrm{mg} / \mathrm{kg}$, sufentanil $0.3 \mu \mathrm{g} / \mathrm{kg}$, and cisatracurium $0.2 \mathrm{mg} / \mathrm{kg}$. Children in Group $\mathrm{F}$ were induced with penehyclidine hydrochloride injection $0.01 \mathrm{mg} / \mathrm{kg}$, propofol $2.0 \mathrm{mg} / \mathrm{kg}$, fentanyl $2 \mu \mathrm{g} / \mathrm{kg}$, and cisatracurium $0.2 \mathrm{mg} / \mathrm{kg}$. When the eyelash reflex was absent, all children were ventilated via a face mask with oxygen $100 \%$. Intubation was performed when spontaneous respiration weakened and a sufficient depth of hypnosis was achieved (lack of palpebral reflex and lack of motoric response to physical stimulation/laryngoscopy). All patients were separately anesthetized with propofol 4-6 $\mathrm{mg} / \mathrm{kg} / \mathrm{h}$ and remifentanil $0.2 \mu \mathrm{g} / \mathrm{kg} / \mathrm{min}$ by an intravenous pump for assisted sedation and assisted analgesia. 
The mechanical ventilation mode was then set to volume control and the tidal volume to $6-8 \mathrm{~mL} / \mathrm{kg}$, and the minute ventilation volume (MV) was adjusted to maintain endtidal $\mathrm{CO}_{2}\left(\mathrm{EtCO}_{2}\right)$ at $35-40 \mathrm{mmHg}$ and the frequency to 18-20 times/min. Single administration of ephedrine 0.5 $1.0 \mathrm{mg} / \mathrm{kg}$ or atropine $0.01 \mathrm{mg} / \mathrm{kg}$ was administered if blood pressure decreased by $30 \%$ of basal values or heart rate was lower than 60 beats $/ \mathrm{min}$. Both the infusion of remifentanil and propofol were discontinued simultaneously at the end of surgery. Neostigmine $0.2 \mathrm{mg} / \mathrm{kg}$ and atropine $0.01 \mathrm{mg} / \mathrm{kg}$ was administered if needed before extubation. After the operation, all the patients were transferred to the post-anesthesia care unit for continuous monitoring.

\section{Data Collection}

\section{Primary Observation Indicators}

Subjective sleep quality was evaluated by the Athens Insomnia Scale (AIS) at the first night before surgery (Sleep preop 1), the first night after surgery (Sleep POD 1), and the third night after surgery (Sleep POD 3). The AIS is a self-rated psychometric questionnaire that quantifies sleep difficulty based on the International Classification of Diseases-10th edition (ICD-10) criteria. It consists of 8 items: sleep induction, waking up during the night, final awakening, total sleep duration, sleep quality, well-being, functioning capacity, and sleepiness during the day. Total AIS scores range from 0 to 24 points, with $\geq 6$ points indicating a diagnosis of insomnia. ${ }^{18}$

\section{Secondary Observation Indicators}

The postoperative pain score was evaluated by the Faces Pain Scale-Revised (FPS-R) $\left(0-10\right.$ points),${ }^{19}$ which is valid for use in children aged 4-12 years to assess acute pain, procedural pain, postoperative pain, and diseaserelated pain. We administered the FPS-R at 2, 4, 6, and 24 hours after surgery. Heart rate (HR) and mean arterial pressure (MAP) were recorded 5 minutes after entering the operation room (T0), at induction (T1), after intubation (T2), when surgery began (T3), when surgery ended (T4), and after extubation (T5). Clinical sedation scales, such as the Observer's Assessment of Alertness and Sedation (OAA/S) scale, have been used as valid tools to assess the level of sedation in children by observing their response to various stimuli, and is assessed at 2 hours after surgery. ${ }^{20}$ An OAA/S score of 3 indicates that the patient "responds only after their name is called loudly/or repeatedly". ${ }^{21}$ For this reason, the OAA/S score 3 was considered as a state of sedation based on clinical observation. Adverse reactions such as postoperative nausea and vomiting (PONV), respiratory depression, hypothermia, agitation, etc. were recorded and treated accordingly.

\section{Statistical Analysis}

SPSS 23.0 (IBM Corp, Armonk, NY, United States) and GraphPad Prism 8.0 statistical software was used for data processing and statistical analysis. The quantitative data of normal distribution are presented as mean \pm standard deviation $(\overline{\mathrm{x}} \pm \mathrm{s})$, and intergroup comparison was performed with independent sample $t$-test. The qualitative data are expressed as number (n) and percentage (\%), and compared with the $\chi^{2}$ test. The linear association between postoperative pain score and sleep quality was analyzed by linear regression analysis to calculate an $r^{2}$ value. $P<0.05$ (two-sided) suggested that the difference was statistically significant.

\section{Results}

As shown in Figure 1, we initially assessed the eligibility of 93, of whom, 11 children did not meet the inclusion criteria and six children's parents refused participation. Finally, 76 children participated in the study. After the study, four patients in Group S were excluded for laryngospasm, hemorrhage, and poor cooperation due to extreme pain and received additional analgesics after surgery. Two patients in Group F were excluded for hemorrhage. Finally, data from 34 patients in Group S and 36 patients in Group F were analyzed.

\section{The Comparison of Demographic Characteristics of the Two Groups}

There were no significant differences between the two groups in terms of age $(\mathrm{P}=0.888)$, sex $(\mathrm{P}=0.611)$, height ( $P=0.722)$, weight $(P=0.205)$, duration of the operation (min) $(\mathrm{P}=0.062)$, and total dose of remifentanil $(\mathrm{mg})(\mathrm{P}=$ 0.005) (Table 1).

\section{Primary Outcomes \\ The Comparison of Postoperative Sleep Quality Between the Two Groups}

As shown in Figure 2B, compared to Sleep preop 1, patients in both groups presented with a lower sleep quality at Sleep POD 1 and Sleep POD 3 (P < 0.001, 


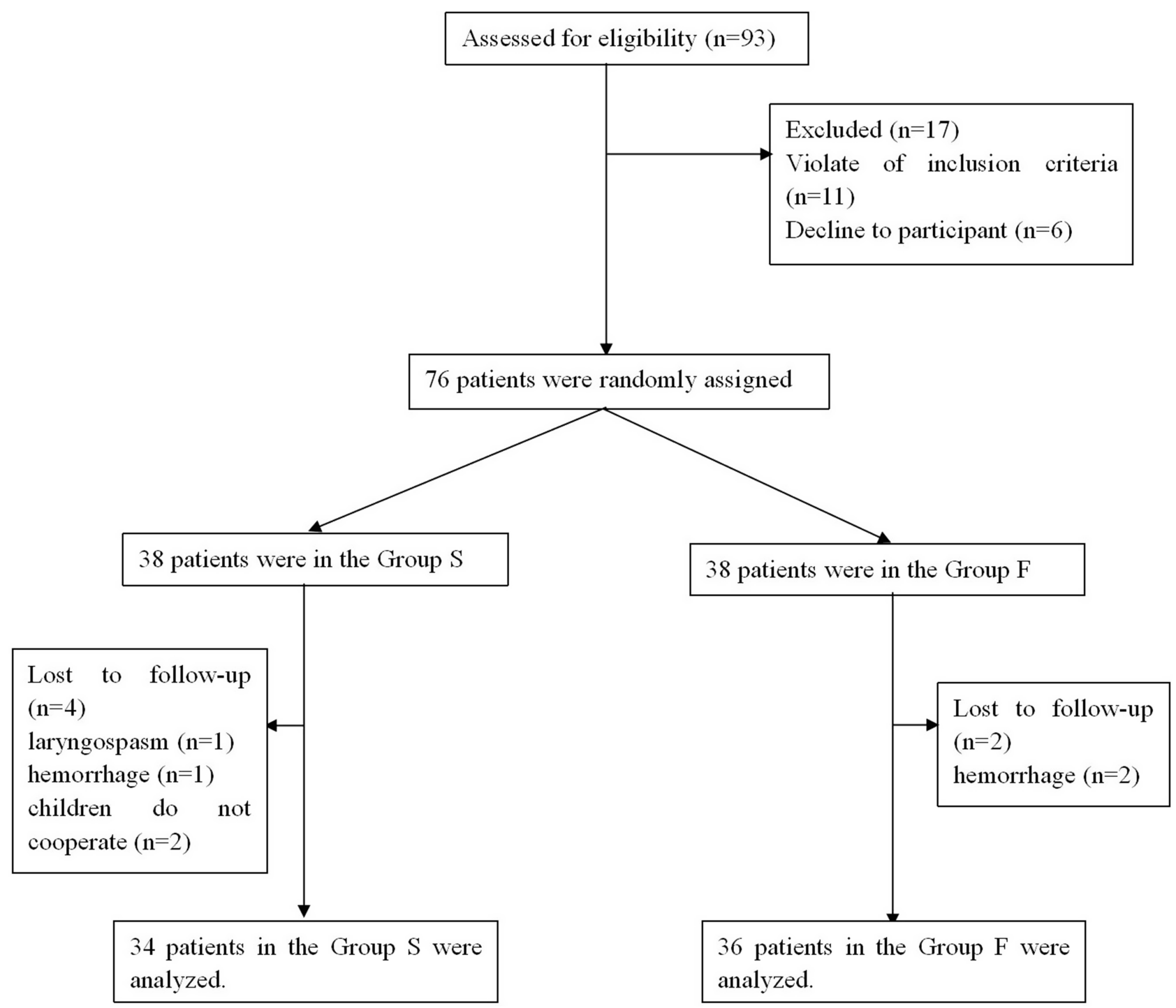

Figure I Flow diagram showing the patients that were included and excluded in this study.

respectively). There was no significant difference in AIS AIS scores, indicating better postoperative sleep quality, score at Sleep preop 1 between the two groups $(\mathrm{P}=$ than patients in Group F at Sleep POD 1 and Sleep POD 0.750). Patients in Group $S$ reported significantly lower $3(\mathrm{P}<0.001$, respectively) (Figure $2 \mathrm{~B})$.

Table I Comparison of Demographic Characteristics Between the Two Groups

\begin{tabular}{|l|l|l|l|}
\hline & Group S (n=34) & Group F $(\mathbf{n = 3 6})$ & P \\
\hline Age (years) & $6.94 \pm 2.7$ & $7.03 \pm 2.5$ & 0.888 \\
Gender (Male/Female) (n) & $14 / 20$ & $17 / 19$ & 0.611 \\
Weight (Kg) & $22.59 \pm 5.5$ & $24.11 \pm 4.4$ & 0.205 \\
Height (cm) & $118.76 \pm 15.6$ & $120.11 \pm 16.0$ & 0.722 \\
Duration of surgery (min) & $86.68 \pm \mid 1.2$ & $80.22 \pm 14.3$ & 0.062 \\
Total does of remifentanil (mg) & $0.32 \pm 0.2$ & $0.41 \pm 0.1$ & 0.005 \\
\hline
\end{tabular}

Notes: variables are presented as mean $( \pm S D)$ or as frequency (percentage). 
A

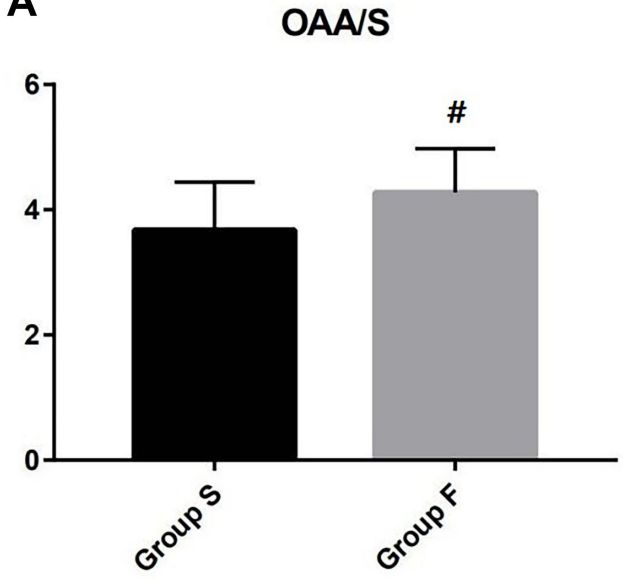

B

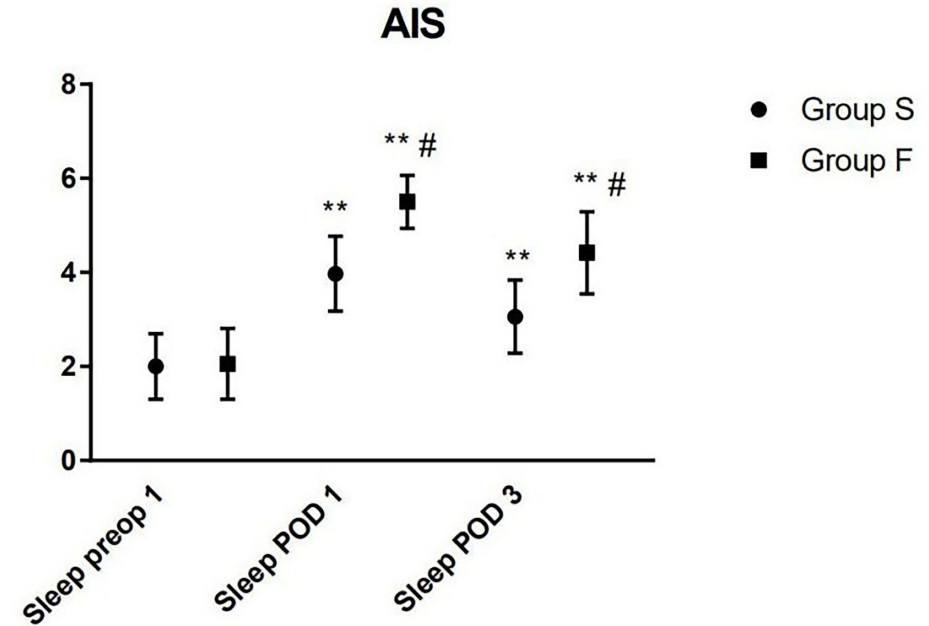

Figure 2 The comparison of postoperative sedation scores and subjective sleep quality between the Group S and the Group F. (A) OAA/S scores; (B) AIS scores. Sleeppreop I: the first night before surgery; Sleep POD I: the first night after surgery; Sleep POD 3: the third night after surgery. **vs the previous time point: $P<0.00$ I. ${ }^{\#}$ vs the Group S: P $<0.001$.

Abbreviations: OAA/S, Observer's Assessment of Alertness and Sedation; AIS, Athens Insomnia Scale.

\section{Secondary Outcomes}

The Comparison of Intraoperative Hemodynamics Between the Two Groups

There was no significant difference in the HR and MAP at T0 between the two groups $(\mathrm{P}=0.455$, and $\mathrm{P}=0.930$, respectively). However, MAP and HR were lower at other time points, from $\mathrm{T} 1$ to $\mathrm{T} 4$, in Group $\mathrm{S}$ compared to Group $\mathrm{F}(\mathrm{P}<0.05$, respectively) (Table 2$)$.

\section{The Comparison of Postoperative Pain, Sedation} Scores and Adverse Effects Between the Two Groups Children in Group S had significantly lower FPS-R scores compared to Group F at 2, 4, and 6 hours after surgery ( $\mathrm{P}$ $=0.004, \mathrm{P}=0.004$, and $\mathrm{P}=0.001$, respectively) (Table 3). The OAA/S score at 2 hours after surgery was lower in Group S compared to Group F (P $<0.001)$ (Figure 2A). There was an approximately linear correlation between the FPS-R $2 \mathrm{~h}$ score and Sleep POD 1. Linear regression analysis revealed an $\mathrm{r}^{2}$ value of $0.081(\mathrm{P}=0.017)$. There was no significant difference in the incidence of PONV between the two groups $(\mathrm{P}=0.435)$. Furthermore, none of children in either group experienced other opioid-related adverse effects such as hypothermia, agitation, and respiratory depression (Table 3).

\section{Discussion}

Our study demonstrated that although postoperative sleep quality was decreased in both groups, children in Group $S$ reported better sleep quality at Sleep POD 1 and Sleep POD 3 than children in Group F. Furthermore, the postoperative pain intensity and sedation scores were lower, and children had better hemodynamic stability during the operation in Group S than in Group F.

Previous studies have proposed a bidirectional model in which higher postoperative pain predicted poorer sleep quality, which in turn predicted higher pain levels on the

Table 2 Comparison of Hemodynamics at Each Time Points Between Two Groups

\begin{tabular}{|l|c|c|c|c|c|c|c|}
\hline Items & Group & T0 & TI & T2 & T3 & T4 & T5 \\
\hline MAP $(\mathrm{mmHg})$ & Group S $(\mathrm{n}=34)$ & $82.21 \pm 10.9$ & $77.18 \pm 8.4$ & $85.12 \pm 10.3$ & $76.85 \pm 8.2$ & $76.06 \pm 8.1$ & $82.18 \pm 6.9$ \\
\cline { 2 - 8 } & Group F $(\mathrm{n}=36)$ & $80.28 \pm 10.6$ & $82.61 \pm 8.9^{*}$ & $89.97 \pm 9.6^{*}$ & $84.69 \pm 10.7^{*}$ & $81.11 \pm 9.6^{*}$ & $86.94 \pm 9.4^{*}$ \\
\hline \multirow{2}{*}{ HR (beat/min) } & Group S $(\mathrm{n}=34)$ & $105.06 \pm 12.7$ & $96.35 \pm 10.1$ & $107.65 \pm 12.5$ & $95.38 \pm 10.2$ & $98.62 \pm 8.3$ & $105.62 \pm 10.7$ \\
\cline { 2 - 8 } & Group F $(\mathrm{n}=36)$ & $104.81 \pm 11.5$ & $102.47 \pm 11.2^{*}$ & $1113.14 \pm 10.1^{*}$ & $102.14 \pm 11.6^{*}$ & $104.19 \pm 9.7^{*}$ & $111.47 \pm 12.7^{*}$ \\
\hline
\end{tabular}

Note: Data are presented as mean ( $\pm \mathrm{SD})$. T0: five minutes after entering the operation room; TI: at the moment of induction; T2: at the moment after intubation; T3: at the moment the surgery began; T4: at the moment the surgery ended; T5: at the moment after extubation. *vs Group S: P < 0.05 .

Abbreviations: MAP, mean arterial pressure; HR, heart rate. 
Table 3 The Comparison of Postoperative Pain and Adverse Effects Between the Group S and the Group F

\begin{tabular}{|l|c|c|c|}
\hline & $\begin{array}{c}\text { Group } \\
\mathbf{S}(\mathbf{n = 3 4 )}\end{array}$ & $\begin{array}{c}\text { Group } \\
\mathbf{F}(\mathbf{n = 3 6})\end{array}$ & $\boldsymbol{P}$ \\
\hline FPS-R scores & & & \\
$2 \mathrm{~h}$ & $3.65 \pm 1.6$ & $4.94 \pm 2.1$ & 0.004 \\
$4 \mathrm{~h}$ & $2.41 \pm 1.5$ & $3.72 \pm 2.0$ & 0.004 \\
$6 \mathrm{~h}$ & $1.47 \pm 1 . \mathrm{I}$ & $2.67 \pm 1.7$ & $0.00 \mathrm{I}$ \\
$24 \mathrm{~h}$ & $0.82 \pm 1.0$ & $1.1 \mathrm{I} \pm 1.2$ & 0.284 \\
\hline Adverse effects & & & \\
Nauseated and vomiting, $n(\%)$ & $2(5.9)$ & $4(1 \mathrm{I} . \mathrm{I})$ & 0.435 \\
Respiratory depression, $n(\%)$ & $0(0)$ & $0(0)$ & - \\
Hypothermia, $\mathrm{n}(\%)$ & $0(0)$ & $0(0)$ & - \\
Agitation, n (\%) & $0(0)$ & $0(0)$ & - \\
\hline
\end{tabular}

Notes: variables are presented as mean $( \pm S D)$ or as frequency (percentage). Abbreviation: FPS-R, Faces Pain Scale Revised.

following day. ${ }^{22,23}$ In our study, compared to Sleep preop 1 , patients in both groups presented with a lower sleep quality at Sleep POD 1 and Sleep POD 3, which may be due to the fact that general anesthesia induces a state of medically low consciousness and is considered to be an independent risk factor for circadian rhythm imbalances and postoperative sleep disturbances. ${ }^{24}$ And there was an approximately linear correlation between the FPS-R $2 \mathrm{~h}$ score and Sleep POD 1, which indicated that high level of postoperative pain predicted worse postoperative sleep quality. Nevertheless, our study found that children in Group F had poorer postoperative sleep quality at Sleep POD 1 and Sleep POD 3 compared to children in Group S. The possible reasons may be as follows: 1) the total dose of remifentanil administered in Group $\mathrm{S}$ was lower than that of Group F, which reduced the effect of opioids on postoperative sleep quality. The use of remifentanil in clinical practice offers several advantages, and it is used for various procedures. However, remifentanil has been consistently linked with the development of opioidinduced hyperalgesia, which may cause several issues, such as delaying recovery after surgery, causing discomfort and higher pain scores, increasing the use of analgesics, and causing other opioid-associated side effects such as decreased sleep quality. ${ }^{25}$ 2) Since the efficacy of sufentanil was better than that of fentanyl, the postoperative pain intensity and sedation scores were better in Group S than Group F, which in turn improved the postoperative sleep quality. In addition, the MAP and HR were lower following the induction of general anesthesia. Studies by De Fátima et al and Sato et al have also shown that opioids could increase vagal tone and decrease sympathetic nervous activity. Propofol may also depress baroreceptor reflex control of HR. The decreases in BP and HR after opioids and propofol may be due to the synergistic action of the two drugs. ${ }^{26,27}$ In our study, children in Group S were induced with $0.3 \mu \mathrm{g} / \mathrm{kg}$ sufentanil. According to Xue et $\mathrm{al}^{28}{ }^{28}$ when used as part of anesthesia induction with propofol and vecuronium in children, the bolus administration of sufentanil $0.3 \mu \mathrm{g} / \mathrm{kg}$ can result in a dose-related attenuation of the cardiovascular intubation response, and can completely abolish the cardiovascular intubation response. Moreover, we found that children induced with sufentanil had better hemodynamic stability, with less fluctuation during the operation than those induced with fentanyl. This could be explained as follows: sufentanil has an 8-10 times higher lipid solubility than fentanyl, a higher affinity for the mu-opioid receptor, and 5-10 times more potency than fentanyl; thus, smaller doses are required to achieve an analgesic and sedative effect. ${ }^{12}$ Monk et al also confirmed that unlike other opioids, sufentanil alone induces significantly less cardiovascular instability. It may cause a reduction in HR and BP shortly after the induction of anesthesia, but the cardiovascular instability usually associated with surgery is largely avoided. ${ }^{13}$ Furthermore, Roberts et al indicated that the opioid-induced PONV risk increases in a dose-dependent manner. ${ }^{29}$ Consistent with their findings, our study suggested that the incidence of PONV in Group $\mathrm{S}$ was lower than that in Group F, although there was no statistical difference. Besides this, none of the children in either group experienced other postoperative adverse effects, including hypothermia, agitation, and respiratory depression.

There are several limitations to our study. Firstly, considering the difficulties in conducting polysomnography in children to assess sleep quality perioperatively, we only collected data on subjective sleep quality using the AIS score in the short-term perioperative period and after surgery. Furthermore, the effect of different opioids on longterm sleep quality after surgery needs to be further studied. Secondly, there are many confounding factors that may affect the quality of postoperative sleep. Although we have tried to reduce the influence of other factors on postoperative sleep quality, such as light, noise, or interference caused by night care, there may have been other unavoidable factors that could have affected our results. Thirdly, we did not evaluate the anxiety and preoperative pain threshold of children in the preoperative period, which 
may affect many parameters such as pain and agitation. Fourth, we only conducted the study in a small sample and at a single center. Thus, the effect of other opioids on postoperative sleep quality and pain in other surgeries under general anesthesia warrants investigation in large scale multicenter studies in the future.

\section{Conclusion}

In conclusion, we demonstrated that children undergoing tonsillectomy and adenotomy after general anesthesia who received sufentanil had better hemodynamic stability intraoperatively, better postoperative sleep quality, and less postoperative pain compared with patients who received fentanyl. Sufentanil should be considered over fentanyl to facilitate rapid recovery in children following tonsillectomy and adenotomy.

\section{Data Sharing Statement}

The individual deidentified participant data in our study could be shared with the readers. Readers can obtain the data by emailing the corresponding author (zhujunchao1@hotmail.com). We did not include specific data and documents from other studies in our study. All the data in our study are available for ten years.

\section{Acknowledgments}

The authors would like to thank Raymond C. Koehler, MD, Ph.D., from the Departments of Anesthesiology and Critical Care Medicine, Johns Hopkins, University, Baltimore, Md, USA and Dr. Weifeng Song, MD, Ph.D., from the Department of Anesthesiology and Perioperative Medicine, School of Medicine, the University of Alabama at Birmingham, Birmingham, Alabama, USA for their discussion and advice on this study.

\section{Author Contributions}

All authors made a significant contribution to the work reported, whether that is in the conception, study design, execution, acquisition of data, analysis and interpretation, or in all these areas; took part in drafting, revising or critically reviewing the article; gave final approval of the version to be published; have agreed on the journal to which the article has been submitted; and agree to be accountable for all aspects of the work.

\section{Funding}

The present study was funded by the Joint plan of key R\&D plan of Liaoning Provincial Science and Technology
Department (2020JH2/10300123), 345 Talent project and the Support Plan for Innovative Talents in Liaoning Higher Education Institution (grant no. 201834).

\section{Disclosure}

The authors report no conflicts of interest in this work.

\section{References}

1. Fortier MA, Chou J, Maurer EL, Kain ZN. Acute to chronic postoperative pain in children: preliminary findings. J Pediatr Surg. 2011;46(9):1700-1705. doi:10.1016/j.jpedsurg.2011.03.074

2. Williams G, Bell G, Buys J, et al. The prevalence of pain at home and its consequences in children following two types of short stay surgery: a multicenter observational cohort study. Paediatr Anaesth. 2015;25(12):1254-1263. doi:10.1111/pan.12749

3. Voepel-Lewis T, Malviya S, Tait AR. A prospective cohort study of emergence agitation in the pediatric postanesthesia care unit. Anesth Analg. 2003;96(6):1625-1630. doi:10.1213/01.ane.0000062522. 21048.61

4. Zieliński J, Morawska-Kochman M, Zatoński T. Pain assessment and management in children in the postoperative period: a review of the most commonly used postoperative pain assessment tools, new diagnostic methods and the latest guidelines for postoperative pain therapy in children. Adv Clin Exp Med. 2020;29(3):365-374. doi:10.17219/acem/112600

5. Many BT, Rizeq YK, Kwon S, et al. Quantifying postoperative sleep loss associated with increased pain in children undergoing a modified Nuss operation. J Pediatr Surg. 2020;55(9):1846-1849. doi:10.1016/ j.jpedsurg.2019.12.003

6. Valrie CR, Bromberg MH, Palermo T, Schanberg LE. A systematic review of sleep in pediatric pain populations. J Dev Behav Pediatr. 2013;34(2):120-128. doi:10.1097/DBP.0b013e31827d5848

7. Tripathi R, Rao R, Dhawan A, Jain R, Sinha S. Opioids and sleep a review of literature. Sleep Med. 2020;67:269-275. doi:10.1016/j. sleep.2019.06.012

8. Taheri R, Seyedhejazi M, Ghojazadeh M, Ghabili K, Shayeghi S. Comparison of ketamine and fentanyl for postoperative pain relief in children following adenotonsillectomy. Pak J Biol Sci. 2011;14 (10):572-577. doi:10.3923/pjbs.2011.572.577

9. Chen F, Wang CY, Zhang J, et al. Comparison of postoperative analgesic effects between nalbuphine and fentanyl in children undergoing adenotonsillectomy: a prospective, randomized, double-blind, multicenter study. Front Pharmacol. 2020;11:597550. doi:10.3389/ fphar.2020.597550

10. Li J, Huang ZL, Zhang XT, et al. Sufentanil reduces emergence agitation in children receiving sevoflurane anesthesia for adenotonsillectomy compared with fentanyl. Chin Med J. 2011;124 (22):3682-3685.

11. Lim L, Jang YE, Kim EH, Lee JH, Kim JT, Kim HS. Comparison of the effects of sufentanil and fentanyl in intravenous patient-controlled analgesia after pediatric moyamoya surgery: a retrospective study. Pediatr Neurosurg. 2020;55(1):36-41. doi:10.1159/000504582

12. Lee HM, Kil HK, Koo BN, Song MS, Park JH. Comparison of sufentanil- and fentanyl-based intravenous patient-controlled analgesia on postoperative nausea and vomiting after laparoscopic nephrectomy: a prospective, double-blind, randomized-controlled Trial. Int J Med Sci. 2020;17(2):207-213. doi:10.7150/ijms.39374

13. Zhang J, Chen L, Sun Y, He W. Comparative effects of fentanyl versus sufentanil on cerebral oxygen saturation and postoperative cognitive function in elderly patients undergoing open surgery. Aging Clin Exp Res. 2019;31(12):1791-1800. doi:10.1007/s40520019-01123-8 
14. Jeon HR, Chae WS, Lee SJ, et al. A comparison of sufentanil and fentanyl for patient-controlled epidural analgesia in arthroplasty. Korean J Anesthesiol. 2011;60(1):41-46. doi:10.4097/kjae.2011.60.1.41

15. Kim DK, Yoon SH, Kim JY, Oh CH, Jung JK, Kim J. Comparison of the effects of sufentanil and fentanyl intravenous patient controlled analgesia after lumbar fusion. J Korean Neurosurg Soc. 2017;60 (1):54-59. doi:10.3340/jkns.2016.0707.007

16. Oh SK, Lee IO, Lim BG, et al. Comparison of the analgesic effect of sufentanil versus fentanyl in intravenous patient-controlled analgesia after total laparoscopic hysterectomy: a randomized, double-blind, prospective Study. Int $J$ Med Sci. 2019;16(11):1439-1446. doi:10.7150/ijms.34656

17. Seemann EA, Willis KD, Mueller ML, et al. Sufentanil versus fentanyl: efficacy and patient satisfaction with intrathecal pain management. Neuromodulation. 2012;15(3):194-199. doi:10.1111/ j.1525-1403.2012.00432.x

18. Soldatos CR, Dikeos DG, Paparrigopoulos TJ. The diagnostic validity of the Athens Insomnia Scale. J Psychosom Res. 2003;55 (3):263-267. doi:10.1016/s0022-3999(02)00604-9

19. Emmott AS, West N, Zhou G, et al. Validity of simplified versus standard self-report measures of pain intensity in preschool-aged children undergoing venipuncture. J Pain. 2017;18(5):564-573. doi:10.1016/j.jpain.2016.12.015

20. Chernik DA, Gillings D, Laine H, et al. Validity and reliability of the observer's assessment of alertness/sedation scale: study with intravenous midazolam. J Clin Psychopharmacol. 1990;10(4):244-251.

21. Park KS, Hur EJ, Han KW, Kil HY, Han TH. Bispectral index does not correlate with observer assessment of alertness and sedation scores during $0.5 \%$ bupivacaine epidural anesthesia with nitrous oxide sedation. Anesth Analg. 2006;103(2):385-389. doi:10.1213/ 01.ane.0000226090.13170.25
22. Bloom BJ, Owens JA, McGuinn M, Nobile C, Schaeffer L, Alario AJ. Sleep and its relationship to pain, dysfunction, and disease activity in juvenile rheumatoid arthritis. $J$ Rheumatol. 2002;29 (1):169-173. doi:10.1007/s10067-013-2409-x

23. Butbul Aviel Y, Stremler R, Benseler SM, et al. Sleep and fatigue and the relationship to pain, disease activity and quality of life in juvenile idiopathic arthritis and juvenile dermatomyositis. Rheumatology. 2011;50(11):2051-2060. doi:10.1093/rheumatology/ker256

24. Krenk L, Jennum P, Kehlet H. Sleep disturbances after fast-track hip and knee arthroplasty. $B r \quad J$ Anaesth. 2012;109(5):769-775. doi:10.1093/bja/aes252

25. Santonocito C, Noto A, Crimi C, Sanfilippo F. Remifentanil-induced postoperative hyperalgesia: current perspectives on mechanisms and therapeutic strategies. Local Reg Anesth. 2018;11:15-23. doi:10.2147/LRA.S143618

26. De F, De Assunção Braga A, Da Silva Braga FS, Potério GM, Filier PR, Cremonesi E. The effect of different doses of propofol on tracheal intubating conditions without muscle relaxant in children. Eur J Anaesthesiol. 2001;18(6):384-388. doi:10.1046/j.0265-0215.2001.00861.x

27. Sato M, Tanaka M, Umehara S, Nishikawa T. Baroreflex control of heart rate during and after propofol infusion in humans. Br J Anaesth. 2005;94(5):577-581. doi:10.1093/bja/aei092

28. Xue FS, Xu YC, Liu Y, et al. Different small-dose sufentanil blunting cardiovascular responses to laryngoscopy and intubation in children: a randomized, double-blind comparison. Br J Anaesth. 2008;100 (5):717-723. doi:10.1093/bja/aen032

29. Roberts GW, Bekker TB, Carlsen HH, Moffatt CH, Slattery PJ, McClure AF. Postoperative nausea and vomiting are strongly influenced by postoperative opioid use in a dose-related manner. Anesth Analg. 2005;101(5):1343-1348. doi:10.1213/01.ANE.0000180204.64588.EC
Nature and Science of Sleep

\section{Publish your work in this journal}

Nature and Science of Sleep is an international, peer-reviewed, open access journal covering all aspects of sleep science and sleep medicine, including the neurophysiology and functions of sleep, the genetics of sleep, sleep and society, biological rhythms, dreaming, sleep disorders and therapy, and strategies to optimize healthy sleep.

\section{Dovepress}

The manuscript management system is completely online and includes a very quick and fair peer-review system, which is all easy to use. Visit http://www.dovepress.com/testimonials.php to read real quotes from published authors. 